\title{
Functional MRI of the immediate impact of transcranial magnetic stimulation on cortical and subcortical motor circuits
}

\author{
Sven Bestmann, ${ }^{1,2}$ Jürgen Baudewig, ${ }^{1}$ Hartwig R. Siebner, ${ }^{3}$ John C. Rothwell ${ }^{2}$ and Jens Frahm ${ }^{1}$ \\ ${ }^{1}$ Biomedizinische NMR Forschungs GmbH am Max-Planck-Institut für biophysikalische Chemie, 37077 Göttingen, Germany \\ ${ }^{2}$ Sobell Department of Motor Neuroscience and Movement Disorders, Institute of Neurology, University College of London, UK \\ ${ }^{3}$ Institute of Neurology, University of Kiel, Germany
}

Keywords: auditory cortex, human, motor cortex, SMA, thalamus

\begin{abstract}
Recent studies indicate that the cortical effects of transcranial magnetic stimulation (TMS) may not be localized to the site of stimulation, but spread to other distant areas. Using echo-planar imaging with blood-oxygenation-level-dependent (BOLD) contrast at 3 Tesla, we measured MRI signal changes in cortical and subcortical motor regions during high-frequency $(3.125 \mathrm{~Hz})$ repetitive TMS (rTMS) of the left sensorimotor cortex (M1/S1) at intensities above and below the active motor threshold in healthy humans. The supraand subthreshold nature of the TMS pulses was confirmed by simultaneous electromyographic monitoring of a hand muscle. Suprathreshold rTMS activated a network of primary and secondary cortical motor regions including M1/S1, supplementary motor area, dorsal premotor cortex, cingulate motor area, the putamen and thalamus. Subthreshold rTMS elicited no MRI-detectable activity in the stimulated $\mathrm{M} 1 / \mathrm{S} 1$, but otherwise led to a similar activation pattern as obtained for suprathreshold stimulation though at reduced intensity. In addition, we observed activations within the auditory system, including the transverse and superior temporal gyrus, inferior colliculus and medial geniculate nucleus. The present findings support the notion that re-afferent feedback from evoked movements represents the dominant input to the motor system via M1 during suprathreshold stimulation. The BOLD MRI changes in motor areas distant from the site of subthreshold stimulation are likely to originate from altered synaptic transmissions due to induced excitability changes in M1/S1. They reflect the capability of rTMS to target both local and remote brain regions as tightly connected constituents of a cortical and subcortical network.
\end{abstract}

\section{Introduction}

Over the last two decades, transcranial magnetic stimulation (TMS) has evolved from a simple method for testing the integrity of the corticospinal pathway to a widely used research tool for probing the function of a variety of areas of the cerebral cortex (for recent reviews see Ilmoniemi et al., 1999; Walsh \& Cowey, 2000; Ziemann \& Rothwell, 2000; Cowey \& Walsh, 2001; Paus, 2002; Siebner \& Rothwell, 2003). The increasing application of TMS, however, is contrasted by the lacunae in our knowledge regarding its mechanisms of action. For example, whereas in the past many studies relied on the assumption that the major impact of TMS on brain activity is focal to the site of stimulation, mounting evidence suggests that TMS can also modulate neural circuits in remote brain regions. In particular, TMS effects in distant areas have been demonstrated by using either TMS alone (Gerschlager et al., 2001; Munchau et al., 2002; Bäumer et al., 2003), or in combination with other mapping techniques such as single-photon emission computed tomography (SPECT) (Okabe et al., 2003), electroencephalography (EEG) (Rossi et al., 2000; Paus et al., 2001b; Strens et al., 2002; Oliviero et al., 2003; Thut et al., 2003), positron emission tomography (PET) (Fox et al., 1997; Paus et al., 1997, 1998, 2001a; Siebner et al., 2000, 2001a,b, 2003a,c; Strafella \&

Correspondence: Dr Sven Bestmann, ${ }^{1}$ Biomedizinische NMR, as above.

E-mail: sbestma@gwdg.de

Received 16 December 2003, revised 19 January 2004, accepted 22 January 2004
Paus, 2001; Chouinard et al., 2003; Lee et al., 2003; Speer et al., 2003a,b; Strafella et al., 2003), and functional magnetic resonance imaging (fMRI) (Bohning et al., 1998, 1999, 2000a,b; Baudewig et al., 2001; Nahas et al., 2001; Bestmann et al., 2003b,c; Kemna \& Gembris, 2003).

The existence of widespread cortical activations within a particular brain system hampers the interpretation of behavioural TMS studies unless the extent of possible TMS effects on brain function is known in sufficient detail. Additional confounds that are barely recognizable without neuroimaging may result in unwanted activations caused by stimulation of brain systems other than the target system, for example involving the auditory, tactile, cutaneous or somatosensory cortex (Paus, 1999; Siebner et al., 1999, 2003c), or cross-modality suppression (Paus, 2000).

Catalysed by recent technical developments in combined TMSfMRI (Shastri et al., 1999; Baudewig et al., 2000; Bestmann et al., 2003a), we used high-frequency repetitive TMS (rTMS) synchronized to echo-planar imaging (EPI) with blood-oxygenation-level-dependent (BOLD) sensitivity in order to investigate cortical and subcortical motor structures during both supra- and subthreshold stimulation of the left primary sensorimotor cortex hand area (M1/S1). Extending previous work at lower field, this high-resolution 3T study further advances the technical aspects of combined rTMS-fMRI and addresses not only primary and secondary motor cortices but also putative modulations in the basal ganglia and thalamus. Moreover, simulta- 
neous recordings of electromyographic activity in the contralateral hand monitored the supra- or subthreshold nature of the used TMS pulses. The results indicate that rTMS of M1/S1 at both intensities can activate distinct cortical and, importantly, subcortical motor circuits, which are not solely attributable to the processing of re-afferent somatosensory feedback. The observation of localized activity changes in response to undesired side-effects of TMS such as auditory and trigeminal stimulation further underscores the use of neuroimaging to disentangle the complex relationship between cortical stimulation and elicited brain activity.

\section{Materials and methods}

\section{Subjects}

Twelve healthy subjects (21-41 years, mean age 27.5 years, seven female) were investigated after giving written informed consent. None of them reported any neuropsychiatric history or any personal or family history of epilepsy. All but one were consistent right-handers according to the Edinburgh inventory (Oldfield, 1971). The experiments were conducted in accordance to the Declaration of Helsinki and local ethics board approval (University of Göttingen).

\section{Experimental protocol}

Each study comprised three successive experimental conditions the order of which was randomised between subjects: (i) rTMS at $3.125 \mathrm{~Hz}$ and $110 \%$ of individual resting motor threshold (RMT); (ii) rTMS at $3.125 \mathrm{~Hz}$ and $90 \%$ of individual active motor threshold (AMT) and (iii) voluntary finger movements requiring dorsi-flexion of the right index finger to functionally locate respective motor regions. Finger movements were acoustically triggered by discharging the TMS coil at the same frequency as during stimulation conditions $(3.125 \mathrm{~Hz})$ but at only $15 \%$ of stimulator output. This intensity is too low to stimulate the cortex (Fisher et al., 2002; Bestmann et al., 2004) but still audible during scanning.

After an initial equilibration period (33.2 s duration corresponding to the acquisition of ten brain volumes) experimental protocols comprised eight epochs of stimulation and rest, that is either rTMS (30 pulses) or voluntary finger movement (9.96 s duration corresponding to three volumes) followed by a period of rest (23.24 s duration corresponding to seven volumes). Accordingly, the total measuring time per paradigm was $4 \mathrm{~min} 59 \mathrm{~s}$. Experimental conditions were separated by five minutes to avoid carry-over effects of the stimulation. Throughout the experiments, subjects were instructed to keep their eyes closed and to relax their hands, unless movement periods were indicated. None of the subjects received more than 800 effective TMS pulses per day, half of them at subthreshold intensity. The study therefore conformed to previously recommended safety guidelines (Wassermann, 1998).

\section{Magnetic resonance imaging}

All experiments were conducted at 3 Tesla (Siemens Trio, Erlangen, effective field strength $2.89 \mathrm{~T}$, resonance frequency $123.234 \mathrm{MHz}$ ). Functional images were acquired with BOLD contrast (Ogawa et al., 1990) using a T2*-weighted single-shot, blipped gradient-echo EPI sequence (frequency-selective fat suppression, TR/TE $=3320 / 36 \mathrm{~ms}$, flip angle $70^{\circ}, 2 \times 2 \mathrm{~mm}^{2}$ in-plane resolution, $4 \mathrm{~mm}$ section thickness). Each volume comprised 20 oblique sections that covered the region from the primary motor cortex to the thalamus and included the superior cerebellar lobule. Anatomical T1-weighted images were acquired in each subject using a high-resolution short-echo time $3 \mathrm{D}$ FLASH sequence $\left(\mathrm{TR} / \mathrm{TE}=11 / 4.92 \mathrm{~ms}\right.$, flip angle $15^{\circ}, 1 \times 1 \mathrm{~mm}^{2}$ resolution, $1 \mathrm{~mm}$ section thickness) covering the whole head.

\section{Transcranial magnetic stimulation}

TMS was accomplished with use of a non-ferromagnetic figure-ofeight coil (70 mm outer wing diameter) connected to a Magstim Rapid stimulator (The Magstim Company, Whitland, Wales, UK). The coil was designed to withstand the mechanical perturbations of a high-field MRI environment. The stimulator generates biphasic electrical pulses of approximately $250 \mu$ s duration and an initial rise time of $50 \mu \mathrm{s}$. The coil was connected to an eight meter long cable and a heat-sensor box outside the magnet room.

According to previously reported procedures (Bestmann et al., $2003 \mathrm{~b}, \mathrm{c}$ ), we used the motor evoked response of the right first dorsal interosseous (FDI) muscle to functionally define the optimal site for rTMS of the left M1/S1. The optimal site at which TMS evoked a maximum motor response in the FDI muscle (referred to as the 'motor hot spot') was marked on the subjects' scalp with a pen. The coil orientation was approximately $45^{\circ}$ away from the medio-lateral axis over the presumed hand area of the precentral gyrus and the initial direction of induced current flow was posterior-to-anterior. Motor thresholds were defined as a percentage of the stimulator output that evoked a clear response in the contralateral target muscle in four out of eight trials either at rest or during $10 \%$ of maximum voluntary contraction. Because the cortical excitability may increase during repetitive stimulation procedures (Pascual-Leone et al., 1994), the stimulation intensity was gradually reduced until rTMS no longer elicited a muscle twitch in the target muscle in two out of four trains of subthreshold rTMS. Thresholds were determined prior to scanning with the subjects outside the MR environment.

\section{Electromyographic recordings}

To further ensure that suprathreshold rTMS did and subthreshold rTMS did not elicit peripheral muscle movements in the contralateral hand, electromyographic (EMG) responses were recorded during scanning from the right FDI muscle with a belly tendon montage using a Brain Amp MR-compatible EEG amplifier (Brain Products, Munich, Germany; sample rate $2000 \mathrm{~Hz}$ ). The non-ferromagnetic sintered $\mathrm{Ag} / \mathrm{AgCl}$ electrodes ( $9 \mathrm{~mm}$ diameter) were connected to an MR-compatible preamplifier. Recordings were fed into a personal computer outside the magnet room via optic fibres. The off-line analysis was performed using Brain Vision 1.0 software (Brain Products). The onset and offset of recordings with MRI gradient artefacts were marked and time-locked averaged to the onset of stimulation epochs. EMG recordings were rectified and low-pass filtered at $50 \mathrm{~Hz}$. At the time of the experiments, a full removal of EPI gradient artefacts, as recently achieved for simultaneous fMRI-EEG recordings (see, for example, Laufs et al., 2003), was not possible. EPI gradient artefacts were therefore minimized by a frequency extraction of the $7-25 \mathrm{~Hz}$ spectral range in EMG recordings. This preserved the temporal information of EMG activity at the expense of some of the quantitative information. Thus, only the occurrence of electromyographic activity during stimulation and movement epochs was inspected, rather than the strength of the electromyographic responses between TMS conditions. Recordings from each session were normalized to the mean EMG amplitude. Paired $t$-tests were used to compare normalized mean EMG levels during TMS and resting epochs.

\section{Combined TMS and fMRI}

As demonstrated in Fig. 1A, a custom-made MR-compatible coil holder made of polyetheretherketon (PEEK) plastic was used for placement and fixation of the TMS coil within the scanner. In all participants, it was possible to place the coil at the previously identified position over the left primary motor cortex. In some cases, it was 


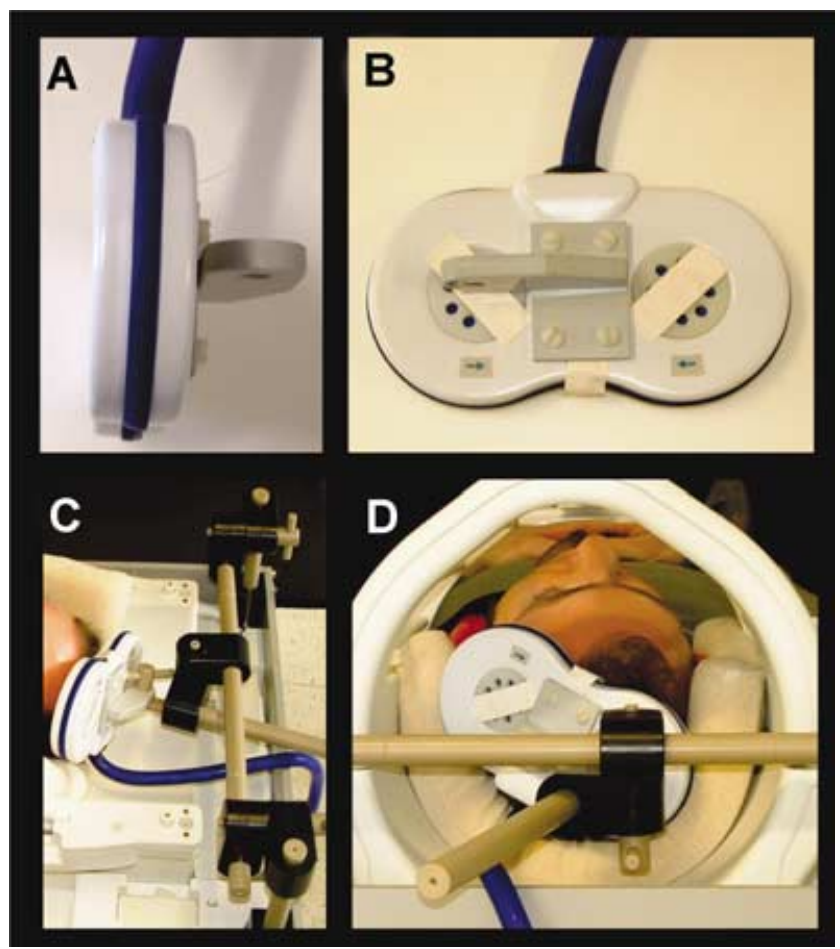

FIG. 1. (A and B) TMS coil. (C) Top-side view of coil holder and attached TMS coil placed over the left M1/S1 with the MRI headcoil open for display purposes. (D) Rear view with TMS coil placed over the left M1/S1 in the closed headcoil.

necessary that subjects slightly tilted their head in order to allow accurate placement of the TMS coil. Head movement was restricted by foam-padded cushions and subjects wore ear-plugs and noise reducing head-phones throughout the entire experiment. Subjects lay supine with their arms rested comfortably onto their sides and near extension of elbows. The good correspondence between the optimal coil position for eliciting a motor evoked potential in contralateral hand muscles and the site of activation within the sensorimotor cortex during voluntary finger tapping (Macdonell et al., 1999; Herwig et al., 2002; Bestmann et al., 2003b) suggests that we successfully targeted the primary sensorimotor hand area. TMS coil position and motor thresholds were briefly evaluated after each experiment by applying single TMS pulses.

The TMS coil was connected to the TMS stimulator outside the radiofrequency $(\mathrm{RF})$ shielded cabin through an RF filter tube. Pilot studies had revealed that the RF emission of the TMS stimulator did not interfere with MRI at 3T, in contrast to previously reported RF interference at 1.5T (see also Bohning et al., 1999; Shastri et al., 1999). This is presumably due to the higher resonance frequency of $123 \mathrm{MHz}$, which is outside the radiofrequency range of common RF sources. Functional images were acquired every $160 \mathrm{~ms}$ with each image acquisition lasting for $91 \mathrm{~ms}$. TMS pulses were applied every $320 \mathrm{~ms}(3.125 \mathrm{~Hz})$, starting immediately after acquisition of a single image, so that the minimum waiting period between a TMS pulse and a subsequent EPI acquisitions was $69 \mathrm{~ms}$. This slightly shorter value than previously recommended for TMS-fMRI at 1.5 and 2.0T (Shastri et al., 1999; Bestmann et al., 2003a,b) was attributed to the improved design and better fixation of the TMS coil, which reduced mechanical vibrations known to induce distorting electromagnetic fields. Because of intersubject anatomical variability of $\mathrm{M} 1$, the corresponding variation of the TMS coil with respect to the $\mathrm{B}_{0}$ field makes it difficult to fully predict and control for torque interactions. In fact, in one subject
EPI images following TMS pulse application were significantly distorted. Noteworthy, this subject had a relatively high motor threshold ( $88 \%$ of stimulator output), so that the corresponding mechanical vibrations may have resulted in electromagnetically induced local field variations lasting longer than the $69 \mathrm{~ms}$ allowed for in the EPI protocol. Hence, the subject was excluded from the study.

In order to avoid damage to the MRI headcoil, direct contact with the TMS coil was strictly avoided, and the induced magnetic field of the TMS coil was never directed directly onto the frame of the headcoil. This prevented the induction of high currents in conductive structures of the MR head coil that might damage functional units, such as the preamplifiers.

\section{Data analysis}

Data analysis and visualization of results were performed using BrainVoyager 2000 (Brain Innovation, Maastricht, the Netherlands). Pre-processing of fMRI acquisitions included temporal filtering (low frequency drift removal), interslice time correction, and motion correction with an exclusion criterion of $>2 \mathrm{~mm}$ in either translation or rotation. Subsequently, 3D functional time series were interpolated into $3 \times 3 \times 3 \mathrm{~mm}^{2}$ spatial resolution and coregistered and transformed into stereotaxic space according to the coordinate system introduced by Talairach \& Tournoux (1988). To facilitate visualization of the results, activation maps were projected onto a template brain provided by the Montreal Neurological Institute (MNI) in stereotaxic space.

Single-subject data was initially analysed by correlation analysis following a previously proposed iterative thresholding procedure (Baudewig et al., 2004). This allowed for a careful inspection of individual images and activation patterns and greatly facilitated an assessment of stimulus-correlated movements or other image distortions. Subsequently, individual preprocessed volume time courses were analysed using the general linear model (GLM). Stimulation or movement epochs were considered as the effects of interest and experimental protocols were convolved with a model of the haemodynamic response function (Boynton et al., 1996) to obtain the respective design matrix. For multisubject analysis we used a fixed effects model to test for significant changes in BOLD signal during each experimental condition at a group level. The significance level for each voxel was set at $P<0.01$ adjusted for multiple comparisons.

In addition, BOLD MRI signal intensity time courses were obtained from a priori anatomically defined regions-of-interest (ROI). These included left (stimulated) and right M1/S1, supplementary motor area (SMA), left and right dorsal premotor cortex (PMd), left and right ventro-lateral nucleus (VLN) of the thalamus, and auditory cortex (AUD). Each ROI was demarcated with a rectangular cube on the respective individual anatomical region without overlap of adjacent ROIs.

M1/S1 was located by the presumed anatomical landmark of the primary motor hand area (White et al., 1997; Yousry et al., 1997). The antero-posterior extent of M1/S1 was designated by the lateral convexity of the pre- and postcentral gyrus, respectively. The PMd was located in the caudal part of the superior frontal gyrus (Fink et al., 1997) extending rostrally to the plane perpendicular to the rostral part of the genu of the corpus callosum. The border between dorsal and ventral premotor cortex is not clearly defined (Grezes \& Decety, 2001) and was marked by the lateral extent of the M1/S1 ROI (Hlustik et al., 2002). Following previous recommendations, the anterior commissure - posterior commissure line was used to demarcate the SMA and the pre-SMA (Picard \& Strick, 1996, 2001). The ventral border was constituted by the cingulate or the paracingulate gyrus (Paus et al., 1996), respectively. The medial ROI (SMA) was collapsed into a single midline ROI not including the pre-SMA or cingulate motor region 
(CMA). The auditory cortex comprised the transverse temporal gyrus (TTG) and the mid-dorsal surface of the superior temporal gyrus. Medially, its extent was restricted by the medial border of the insula. The caudal border was marked by the extent of the TTG, while the rostral extent was arbitrarily confined by the level of the central sulcus. As the ventro-lateral nucleus of the thalamus (VLN) cannot easily be distinguished from other thalamic nuclei on T1-weighted images, it was defined by a region starting at the level of the posterior commissure and extending rostrally halfway to the anterior commissure, and from the lateral border of the thalamus to halfway its medial border.

Mean MRI signal intensity time courses were expressed as percentage change with reference to the last three time points of each experimental cycle. Signal time courses were time-locked averaged for each ROI. Mean signal intensities were calculated for each functional image in each ROI.

\section{Results}

None of the subjects reported any side-effects from the experimental procedure apart in some cases from slight discomfort on the head resulting from the pressure of the TMS coil. In one subject, no electromyographic responses could be recorded due to a malfunction of the recording computer. However, as recordings from all other subjects confirmed the presence or absence of EMG responses to supra- and subthreshold stimulation, respectively, the data from this subject was not excluded from the final analysis.

\section{Motor system}

As can be seen in Fig. 2 and similar to voluntary finger dorsi-flexion (not shown), suprathreshold rTMS evoked clear electromyographic activity in the right FDI muscle when compared to resting epochs ( $t$ test, $P<0.01)$. In contrast, no differences in EMG activity during subthreshold rTMS and baseline epochs was found ( $t$-test, $P=0.22$ ), thus confirming its subthreshold nature.

Individual activation maps superimposed onto EPI raw images together with the corresponding unthresholded correlation maps are shown in Fig. 3 for a representative subject and two brain sections. The examples refer to voluntary finger movement as well as rTMS at supraand subthreshold intensity. These images clearly demonstrate the absence of image artefacts related to simultaneous rTMS, confirming that we had allowed for sufficient waiting periods between TMS pulses and MR image acquisition.

Figure 4 illustrates the results of a group analysis by showing statistically significant activations evoked by suprathreshold rTMS as projections onto a segmented reference brain (MNI). Quantitative information about respective centres of gravity (in Talairach coordinates), $t$-values, and cluster volumes are summarized in Table 1. At a group level, focal increases in synaptic activity (as indexed by an increase in BOLD signal) was detected in the stimulated left M1/S1 hand area, PMd and the inferior part of the precentral gyrus (putative PMv), bilateral SMA and CMA, and to a lesser extent, the right PMd. The left cortical motor-related activation on the lateral surface extended from $y=-9 \mathrm{~mm}$ to $-29 \mathrm{~mm}$. In the midline region, activation in the SMA spanned from $y=-16 \mathrm{~mm}$ to $14 \mathrm{~mm}$. Furthermore, there was localized activity in the region around the left ventral posterior middle temporal gyrus (mean coordinates $x=-49$, $y=-51, z=7$ ). The homologous of the right M1/S1 contralateral to the site of stimulation showed a significantly decreased BOLD MRI signal. Activity in the ipsilateral (right) antero-dorsal cerebellar lobule was demonstrated in eight subjects (mean coordinates $x=12, y=-46$, $z=-12)$.
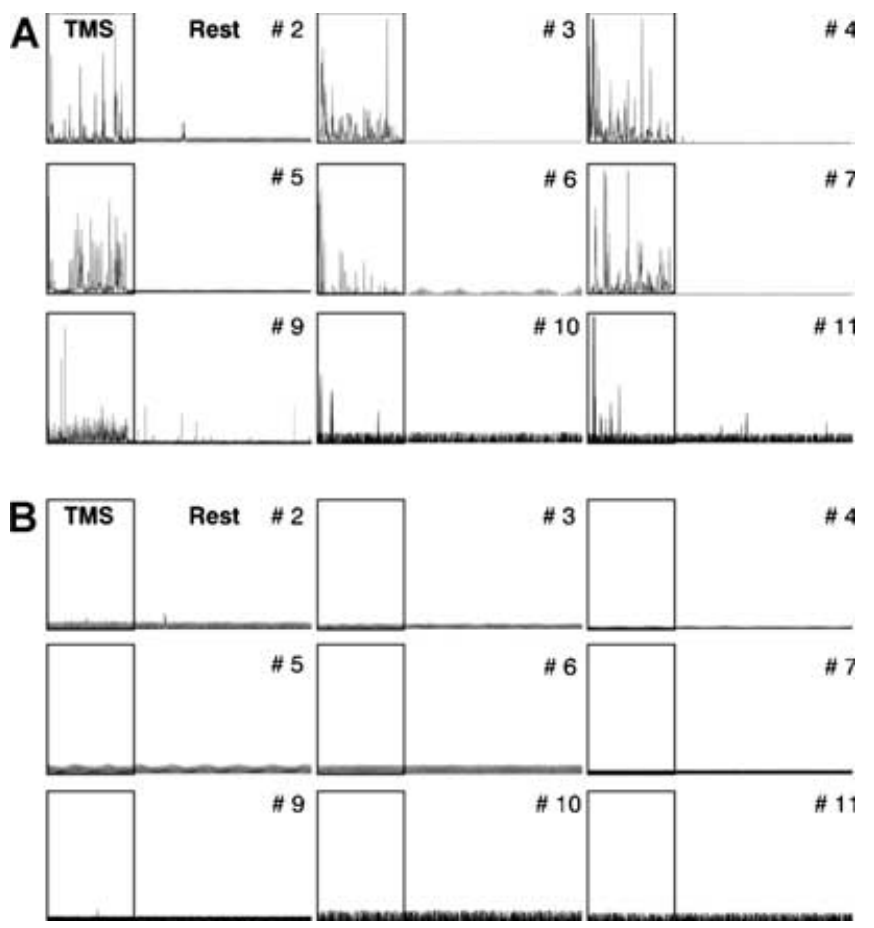

FIG. 2. Electromyographic recordings of (A) suprathreshold and (B) subthreshold rTMS from nine subjects during fMRI. Stimulation epochs (boxes) were time-locked averaged to the onset of stimulation. Frequency extraction (7$25 \mathrm{~Hz}$ ) was performed to reduce MRI gradient artefacts. In comparison to resting epochs, suprathreshold rTMS evoked clear electromyographic activity in the contralateral FDI muscle $(P<0.01)$, whereas no such activity was observed during subthreshold rTMS $(P=0.22)$.

At the subcortical level, Fig. 5 shows selected individual activation maps of all nine subjects with thalamic activations in response to suprathreshold rTMS. In all cases, the centre of gravity was located at the presumed coordinates of the left VLN. Less consistent and much smaller activity was located in the right VLN. Activity of the lentiform nucleus was primarily located in the ventro-medial postcommissural portion of the putamen, although such findings were characterized by considerable interindividual variability. A corresponding group analysis is shown in Fig. 6 depicting enlarged views in all three orientations.

\section{Auditory system}

In addition to motor activity, Fig. 4 shows widespread bilateral activity within the primary and secondary auditory cortex, the superior and dorsal part of the middle temporal gyrus, the planum temporale, and the depth of the Sylvian fissure covering the entire TTG. Again, Talairach coordinates of centres of gravity and activation volumes are given in Table 1. There was considerable overlap in antero-posterior extent (left, $y=5 \mathrm{~mm}$ to $-45 \mathrm{~mm}$; right, $y=-12 \mathrm{~mm}$ to $-41 \mathrm{~mm}$ ) and medio-lateral extent of activated regions in the temporal lobe (left, $x=-64 \mathrm{~mm}$ to $-29 \mathrm{~mm}$; right, $x=-64 \mathrm{~mm}$ to $31 \mathrm{~mm}$ ). As shown in Fig. 4C, the left-sided temporal cluster extended more rostrally and fused with a cluster in the inferior part of the precentral sulcal region. At the subcortical level shown in Figs 5 and 6, bilateral activation was found in the inferior colliculi and the medial geniculate nucleus (MGN), which tended to be lateralized to the left.

\section{Subthreshold rTMS vs. suprathreshold rTMS}

Subthreshold rTMS did not yield significant activations in the stimulated M1/S1, but caused marked BOLD MRI signal changes in the 


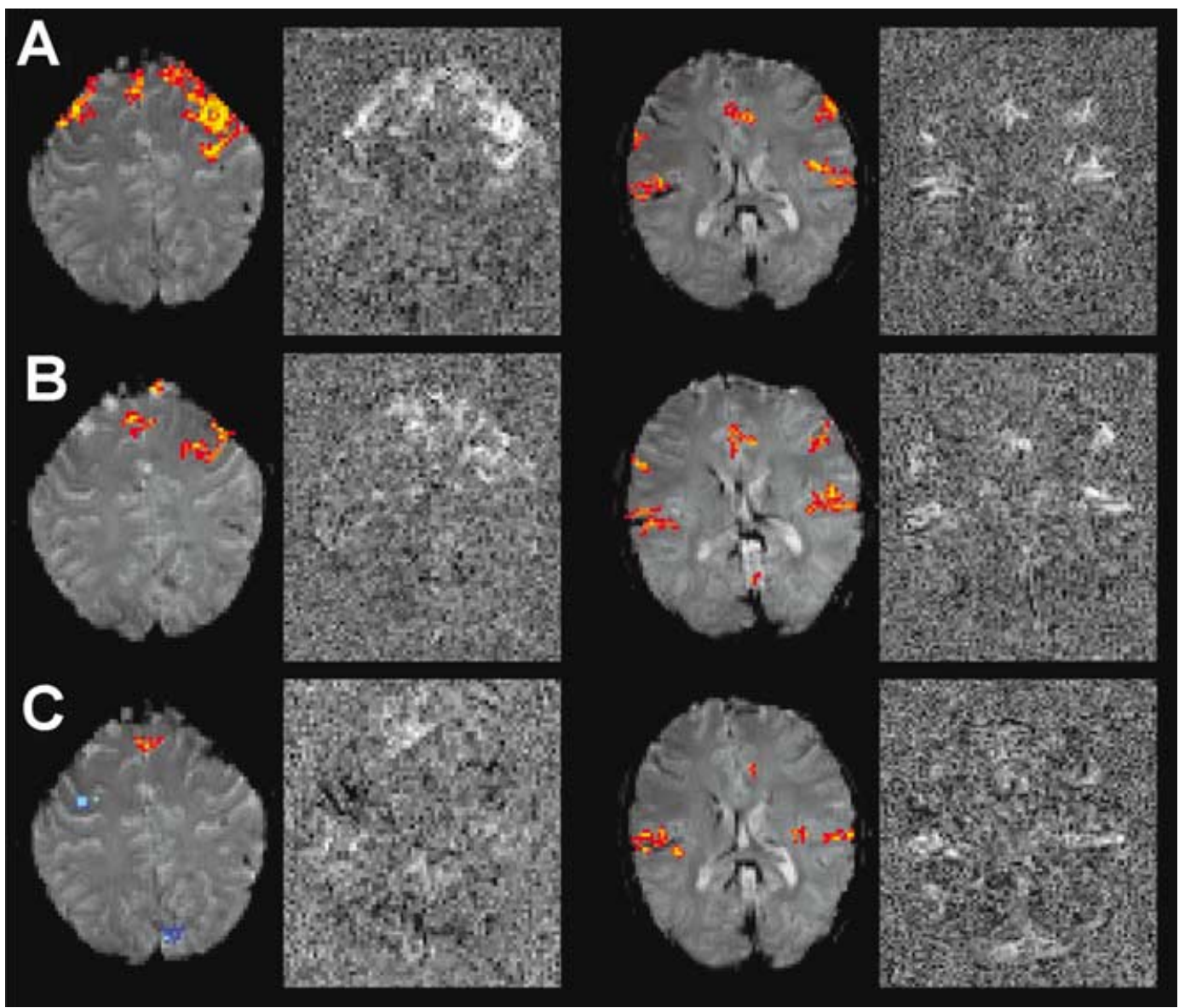

FIG. 3. Individual activation maps superimposed onto EPI raw images and corresponding unthresholded correlation maps from a representative subject obtained for (A) an acoustically cued voluntary finger movement (B) suprathreshold rTMS at $110 \%$ resting motor threshold (RMT), and (C) subthreshold rTMS at $90 \%$ active motor threshold (AMT) in oblique sections cutting through (left) the M1/S1 and (right) the auditory cortex. While activations, that is positive BOLD MRI responses or signal increases, are coded in red-yellow, BOLD MRI signal decreases are coded in blue. No image degradation was revealed during rTMS at any intensity.

SMA, CMA, and left PMd. Additional activations were seen in the left and right ventro-lateral thalamus. No consistent activity changes were detected in the striatum. These findings are visualized in Fig. 7 comparing subthreshold rTMS with suprathreshold rTMS, while quantitative data for subthreshold rTMS are given in Table 2 . In the auditory cortex, subthreshold rTMS elicited widespread activations similar to those found for suprathreshold rTMS, ranging from $y=-18 \mathrm{~mm}$ to $-42 \mathrm{~mm}$ (left) and $y=-9 \mathrm{~mm}$ to $-40 \mathrm{~mm}$ (right). In the group analysis, significant activation in the inferior colliculus was only detected in the left hemisphere. Figure 7 further demonstrates that BOLD MRI signal decreases were found for both TMS conditions not only in the right M1/S1 contralateral to the site of stimulation and the medial paracentral lobule, but also in the occipital and posterior parietal cortex, with a right-hemispheric preponderance. These latter decreases were located in the medial occipital cortex (putative V1), right transverse occipital sulcus (putative V5), and posterior parietal cortex.

Figure 8 summarizes BOLD MRI signal intensity time courses obtained from six regions-of-interest and for all three experimental conditions, that is voluntary finger movement as well as supra- and subthreshold rTMS. With the exception of the stimulated M1/S1, all predefined regions-of-interest showed a significant modulation of the regional BOLD signal in response to subthreshold rTMS. In the stimulated M1/S1, a modulation of the BOLD signal occurred only during suprathreshold rTMS. No differences in signal intensity were seen in the auditory cortices and left thalamus.
As shown in Fig. 9 and summarized in Table 3, a direct comparison of activations evoked by supra- and subthreshold rTMS yielded significant differences in several cortical motor regions, while no differences were found in auditory cortical or subcortical areas. In more detail, suprathreshold rTMS led to stronger activations in the medial cortical regions of the left M1/S1 hand area, the SMA and CMA, the left PMd, and left parietal operculum. Furthermore, no significant difference in auditory activity was detected when rTMS was contrasted with voluntary finger movement (not shown). Voluntary finger movement elicited significantly stronger responses in all motor regions when compared to either supra- or subthreshold rTMS.

\section{Discussion}

The present results demonstrate for the first time that activations of distinct motor networks by short periods of focal rTMS of the sensorimotor cortex can be detected with BOLD-sensitive fMRI at a field strength of 3T. In particular, the underlying changes of the BOLD MRI signal were not localized to the area stimulated by TMS but involved a range of cortical and subcortical motor pathways and, furthermore, cover major parts of the auditory system. While the activations of multiple motor areas illustrate the capability of TMS to act on functionally and anatomically connected circuits remote to the stimulated brain region, the occurrence of auditory activations emphasizes the requirement to carefully control for the confounding influ- 


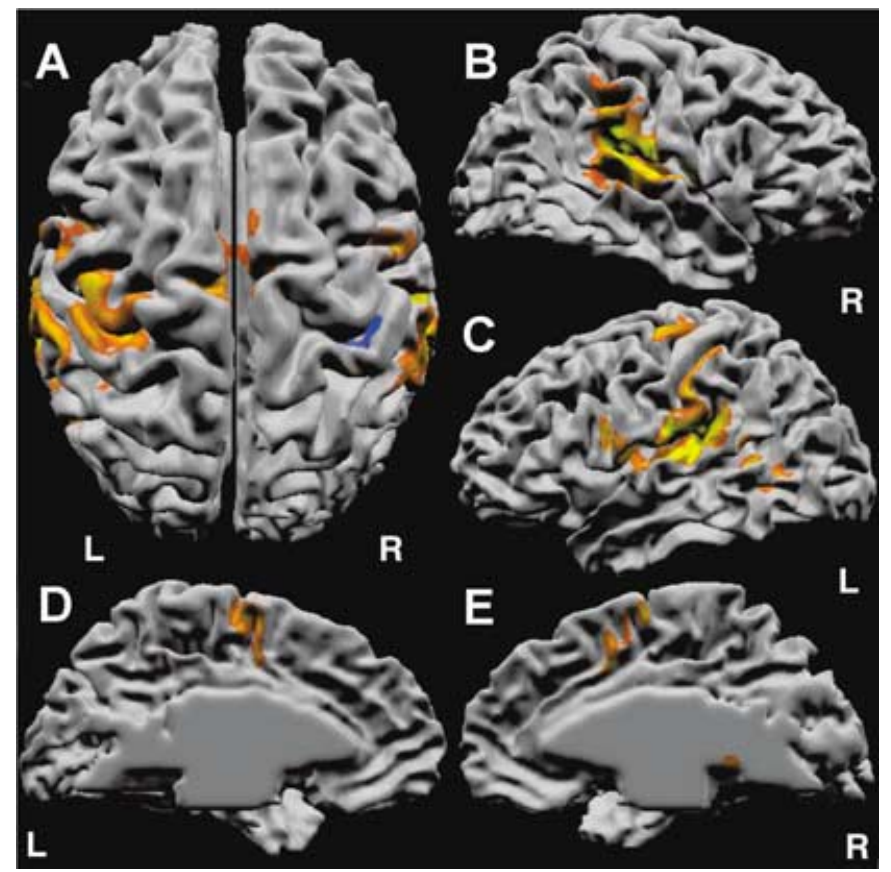

FIG. 4. Cortical activations obtained for a group analysis ( 11 subjects, $P<0.01$, corrected) of responses to suprathreshold rTMS. The maps represent (A) dorsal (B and C) lateral, and (D and E) medial views of significant activations projected onto a 3D surface reconstruction of a template brain (Montreal Neurological Institute, MNI). Increased activity (red-yellow) was found in left M1/S1, PMd, bilateral SMA and auditory cortices, postcentral sulcus, and left ventral posterior middle temporal gyrus. In the left hemisphere, additional activity was found along the inferior precentral sulcus. Activations in the putamen and thalamus are not shown on these 3D views. Decreased BOLD MRI signal intensities (blue) were found in the right M1/S1 hand region and the occipital cortex (not shown). L, left; R, right. ences on regional synaptic activity due to concomitant sensory stimulation.

\section{Motor system}

The extent of TMS-induced activity changes within cortical and subcortical motor networks was similar to that observed previously using PET (Paus et al., 1997, 1998; Siebner et al., 2000, 2001a,b, 2003a; Lee et al., 2003) or fMRI (Nahas et al., 2001; Bestmann et al., 2003b). Apart from the contralateral M1/S1, pertinent areas include the left dorsal premotor area together with bilateral posterior SMA and CMA. Subcortical activations were seen in ipsilateral cerebellum, ventrolateral thalamus and ventro-medial putamen. Because similar activation patterns are elicited in response to voluntary finger movements, we presume that much of this activity reflects the anatomical connectivity of the stimulated M1/S1 known to possess direct monosynaptic connections with all of the aforementioned areas. This understanding is also consistent with the fact that TMS activated the posterior part of the SMA rather than the pre-SMA. Whereas the former is intimately linked with M1 (Geyer et al., 2000), the latter predominantly projects to the prefrontal cortex and does not have any direct connections with M1/S1 (Rizzolatti et al., 1996).

In general, the pattern of changes in activity within the motor system was similar during suprathreshold and subthreshold rTMS except for the directly stimulated M1/S1, which was only activated during suprathreshold stimulation. As suggested previously, the most likely explanation for this difference is due to the fact that much of the suprathreshold M1/S1 activation represents the afferent feedback from the actual muscle movements induced by the TMS pulses (Baudewig et al., 2001; Bestmann et al., 2003b,c). Nevertheless, the complete absence of any BOLD MRI signal changes in M1/S1 during subthreshold TMS is surprising for two reasons. First, there is good electrophysiological evidence that subthreshold stimulation activates the cortical circuitry even if it fails to elicit the corticospinal output

TABLE 1. Activations evoked by suprathreshold rTMS (110\% resting motor threshold)

\begin{tabular}{|c|c|c|c|c|c|}
\hline \multirow[b]{2}{*}{ Anatomical/functional location } & \multicolumn{3}{|c|}{ Talairach coordinates* } & \multirow{2}{*}{$\begin{array}{l}\text { Peak }^{\dagger} \\
\text { activation } \\
t \text {-value }\end{array}$} & \multirow{2}{*}{$\begin{array}{l}\text { Volume } \\
\left(\mathrm{mm}^{3}\right)\end{array}$} \\
\hline & $x$ & $y$ & $z$ & & \\
\hline Left central sulcus (M1/S1) & -36 & -22 & 56 & 7.86 & 1600 \\
\hline Left precentral sulcus (PMd) & -28 & -15 & 65 & 6.04 & 267 \\
\hline Left inf. precentral sulcus (PMv) & -52 & 0 & 17 & 10.52 & 1806 \\
\hline Left postcentral sulcus & -56 & -25 & 43 & 7.80 & 997 \\
\hline Left auditory cortex & -47 & -29 & 17 & 16.28 & 7346 \\
\hline Left ventral posterior middle temp. gyrus & -49 & -51 & 7 & 6.42 & 493 \\
\hline Left insular cortex & -35 & -1 & 15 & 10.36 & 964 \\
\hline Left ventro-lateral thalamus & -11 & -14 & 11 & 9.33 & 966 \\
\hline Left putamen & -27 & -7 & 12 & 7.61 & 590 \\
\hline L. inferior colliculus/Medial geniculate $n$. & -6 & -26 & -2 & 6.35 & 185 \\
\hline Supplementary motor area (SMA) & 1 & -10 & 60 & 9.58 & 1327 \\
\hline Cingulate motor area (CMA) & 1 & 1 & 41 & 9.14 & 1365 \\
\hline Posterior supplementary motor area & 1 & -36 & 56 & -7.13 & 245 \\
\hline Right central sulcus (M1/S1) & 39 & -29 & 54 & -5.86 & 276 \\
\hline Right auditory cortex & 49 & -29 & 16 & 12.97 & 6883 \\
\hline Right ventro-lateral thalamus & 11 & -12 & 10 & 6.86 & 188 \\
\hline Right inferior colliculus & 4 & -26 & -6 & 6.30 & 119 \\
\hline Right posterior parietal cortex & 17 & -83 & 24 & -5.31 & 386 \\
\hline Right transverse occipital sulcus & 29 & -82 & 13 & -5.77 & 189 \\
\hline Right cerebellar hemisphere & 12 & -46 & -12 & 11.97 & 1122 \\
\hline
\end{tabular}

${ }^{*}$ Talairach coordinates correspond to the centre of gravity of respective activation clusters. ${ }^{\dagger}$ Peak activation within cluster $(P<0.01$, corrected $)$. Volumes are given in voxel sizes of $1 \times 1 \times 1 \mathrm{~mm}^{3}$. 


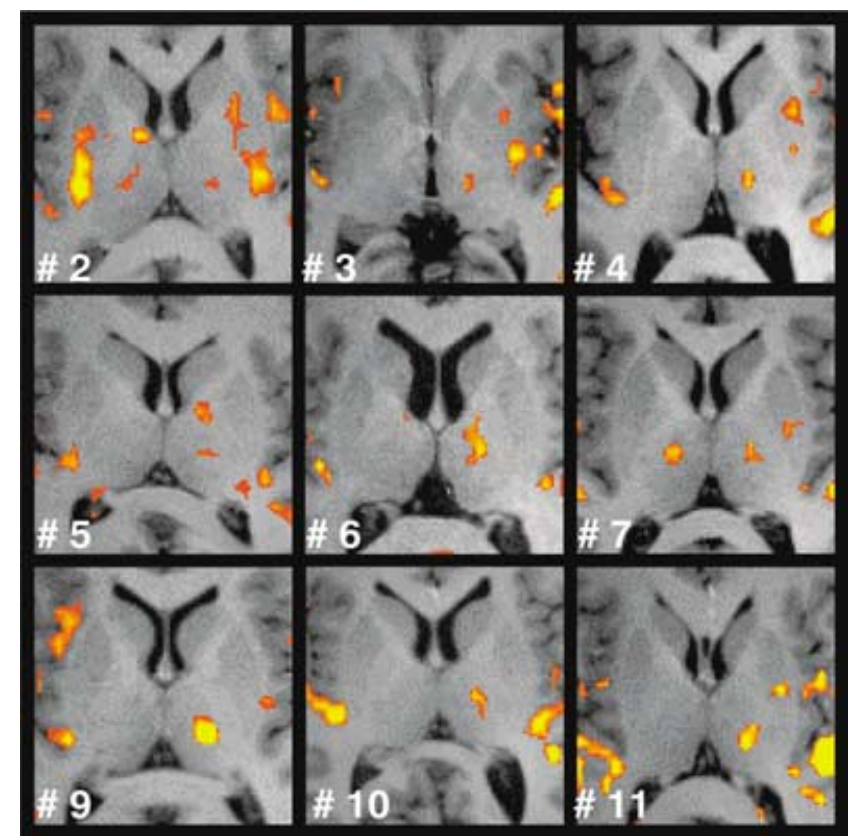

Fig. 5. Individual activation maps of the nine subjects who presented with lefthemispheric thalamic activity in response to suprathreshold rTMS $(P<0.0001$, uncorrected) superimposed onto individual anatomical images in stereotaxic space $(z=9)$. Several subjects showed additional activity in the putamen and/or right thalamus (not always visible in the selected sections).

necessary for a muscle twitch (Di Lazzaro et al., 1998; Fisher et al., 2002). Recordings from implanted spinal epidural electrodes probing descending motor activity indeed demonstrated that little if any activity is evoked by TMS below active motor threshold (Di Lazzaro

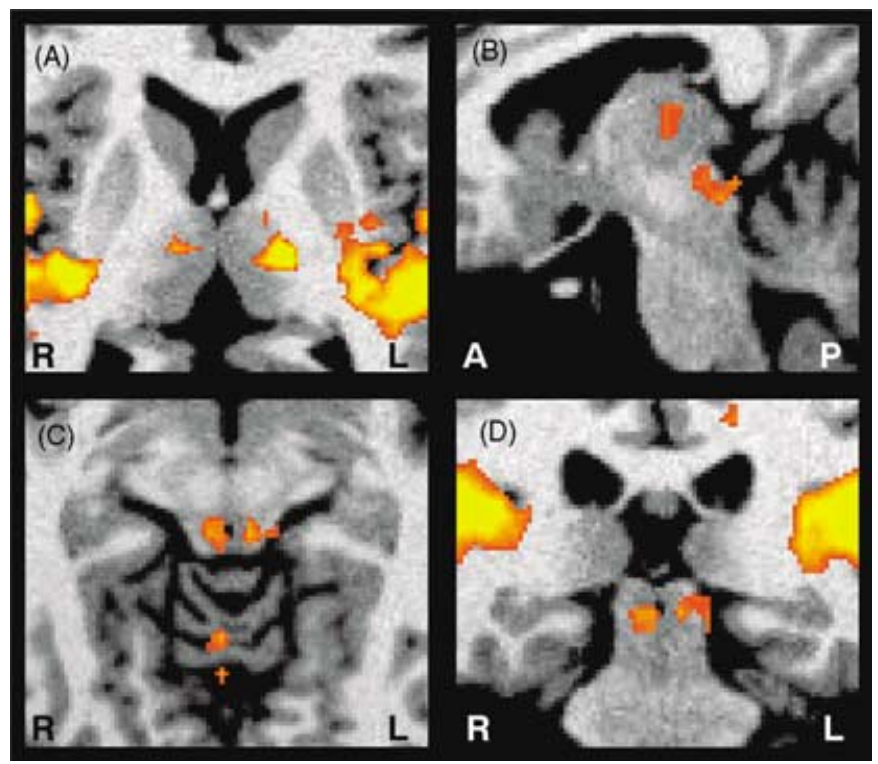

Fig. 6. Subcortical activations obtained for a group analysis (11 subjects, $P<0.01$, corrected) of responses to suprathreshold rTMS. (A) Enlarged view of a transverse section $(z=9)$ of a standard reference brain (MNI) with pronounced activation of the postero-commissural left ventro-lateral thalamus and putamen as well as smaller activations in the right thalamus contralateral to the stimulation. (B) Sagittal section $(x=-5)$ demonstrating distinct activations in the motor and auditory regions of the thalamus. (C) Transverse section $(z=-5)$ with bilateral activation of the inferior colliculi. (D) Coronal section $(y=-26)$ showing bilateral activation of the inferior colliculi. Note also the pronounced activation in the entire auditory cortex. L, left; R, right.

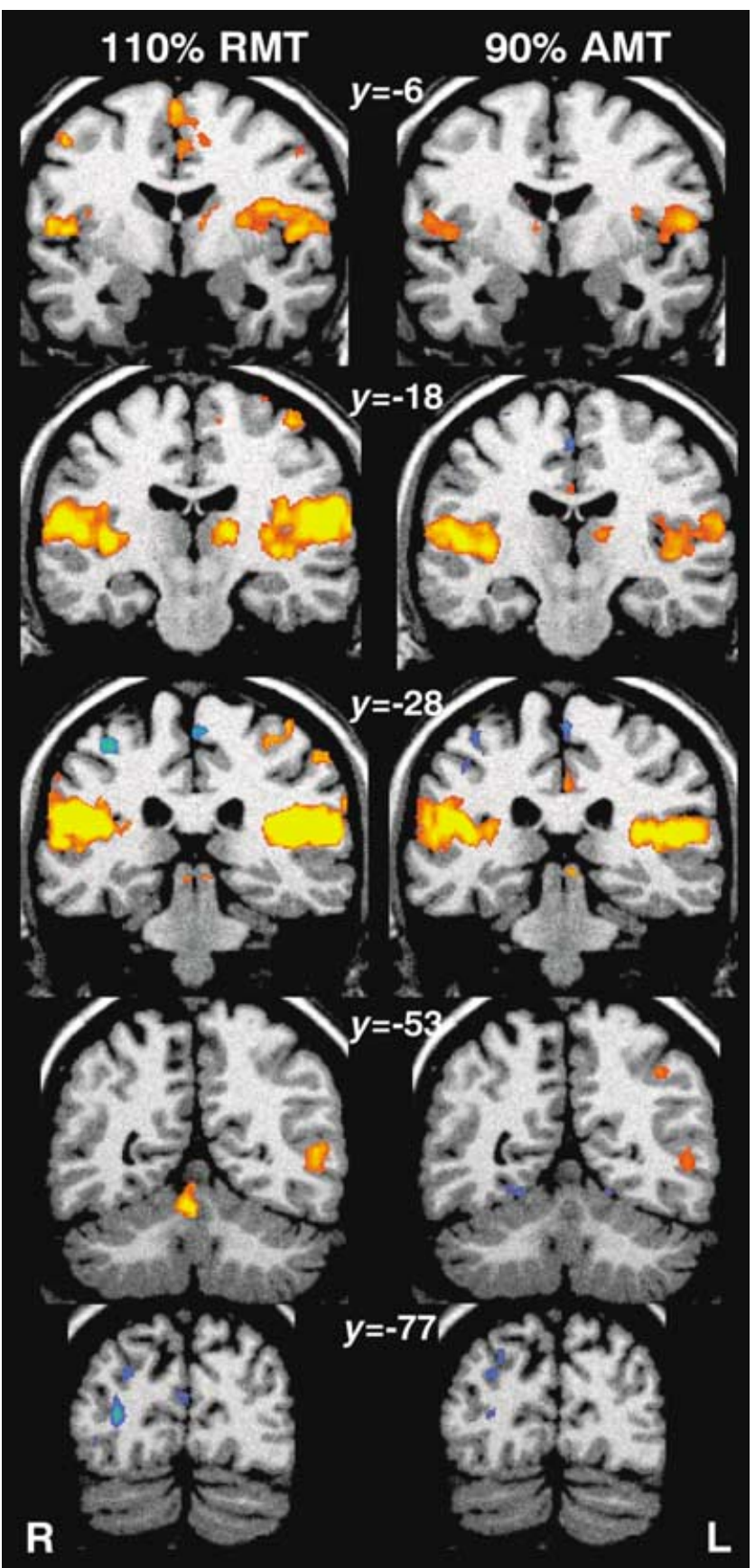

FIG. 7. Activations obtained for a group analysis ( 11 subjects, $P<0.01$, corrected) of responses to (left) suprathreshold rTMS at $110 \%$ resting motor threshold (RMT), and (right) subthreshold rTMS at $90 \%$ active motor threshold (AMT) projected onto five coronal sections of a standard reference brain (MNI, Talairach coordinates indicated). Apart from pronounced auditory activation, suprathreshold rTMS induced activations (red-yellow) in the left M1/S1, the medial SMA and cingulate motor region, the lateral postcentral region (putative S1 and S2) and the left thalamus. BOLD MRI signal decreases (blue) were observed in the right M1/S1 and occipital cortex. Except for the stimulated left M1/S1, subthreshold rTMS evoked similar but smaller activations.

et al., 1998, 2002). On the other hand, paired pulse experiments in which a small initial stimulus is used to condition the response to a larger test stimulus clearly show that a subthreshold TMS pulse can affect the excitability of cortical circuits (Kujirai et al., 1993; Fisher et al., 2002; Bestmann et al., 2004). Pharmacological evidence suggests that much of this altered excitability is synaptically mediated via 
TABLE 2. Activations evoked by subthreshold rTMS (90\% AMT)

\begin{tabular}{|c|c|c|c|c|c|}
\hline \multirow[b]{2}{*}{ Anatomical/functional location } & \multicolumn{3}{|c|}{ Talairach coordinates ${ }^{*}$} & \multirow{2}{*}{$\begin{array}{l}\text { Peak }^{\dagger} \\
\text { activation } \\
t \text {-value }\end{array}$} & \multirow{2}{*}{$\begin{array}{l}\text { Volume } \\
\left(\mathrm{mm}^{3}\right)\end{array}$} \\
\hline & $x$ & $y$ & $z$ & & \\
\hline Left precentral sulcus (PMd) & -26 & -14 & 62 & 5.04 & 108 \\
\hline Left auditory cortex & -46 & -24 & 13 & 13.82 & 6039 \\
\hline Left ventro-lateral thalamus & -10 & -14 & 11 & 6.74 & 599 \\
\hline Left inf. precentral gyrus (PMv) & -52 & -2 & 14 & 8.49 & 1157 \\
\hline L. inferior colliculus/Medial geniculate $n$. & -5 & -25 & -3 & 4.51 & 82 \\
\hline Supplementary motor area (SMA) & 5 & -5 & 59 & 5.68 & 284 \\
\hline Cingulate motor area (CMA) & 2 & 8 & 42 & 5.60 & 515 \\
\hline Posterior supplementary motor area & 0 & -30 & 57 & -7.15 & 450 \\
\hline Medial occipital cortex & 1 & -82 & 14 & -6.32 & 440 \\
\hline Right central sulcus (M1/S1) & 35 & -30 & 53 & -7.26 & 321 \\
\hline Right auditory cortex & 49 & -21 & 14 & 12.09 & 5280 \\
\hline Right ventro-lateral thalamus & 11 & -11 & 11 & 6.86 & 159 \\
\hline Right posterior parietal cortex & 14 & -84 & 29 & -7.30 & 199 \\
\hline Right transverse occipital sulcus & 30 & -79 & 9 & -7.77 & 310 \\
\hline
\end{tabular}

${ }^{*}$ Talairach coordinates correspond to the centre of gravity of respective activation clusters. ${ }^{\dagger}$ Peak activation within cluster $(P<0.01$, corrected). In voxel sizes of $1 \times 1 \times 1 \mathrm{~mm}^{3}$.
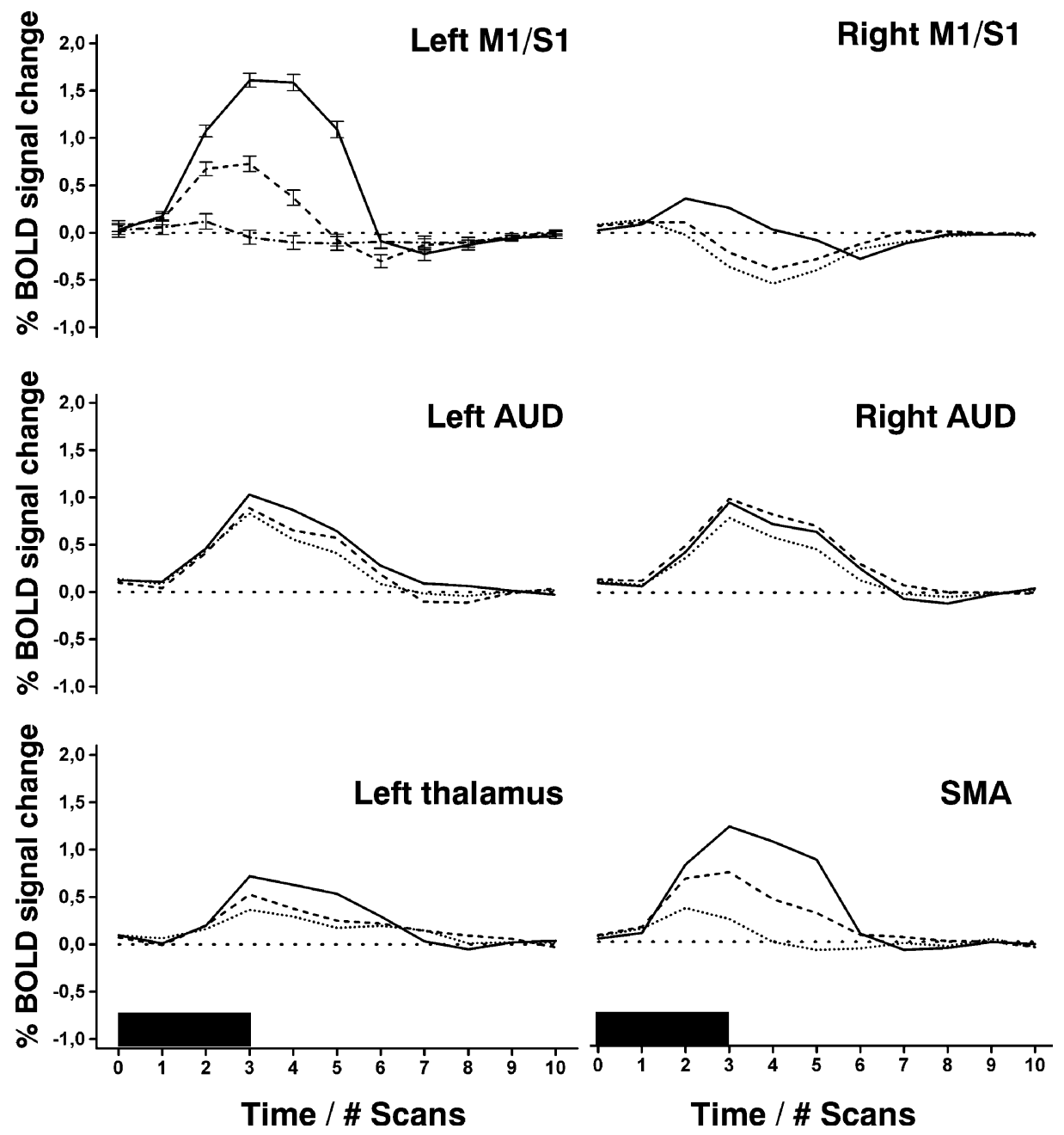

FIG. 8. BOLD MRI signal intensity time courses (mean \pm SEM, only indicated in the upper left graph for the sake of visibility) from six regions-of-interest for voluntary finger movement (solid line), suprathreshold rTMS at 110\% resting motor threshold (dashed line), and subthreshold rTMS at $90 \%$ active motor threshold (dotted line). Stimulation periods are indicated by black bars. M1/S1, sensorimotor cortex; PMd, dorsal premotor cortex; SMA, supplementary motor area; AUD, auditory cortex. 


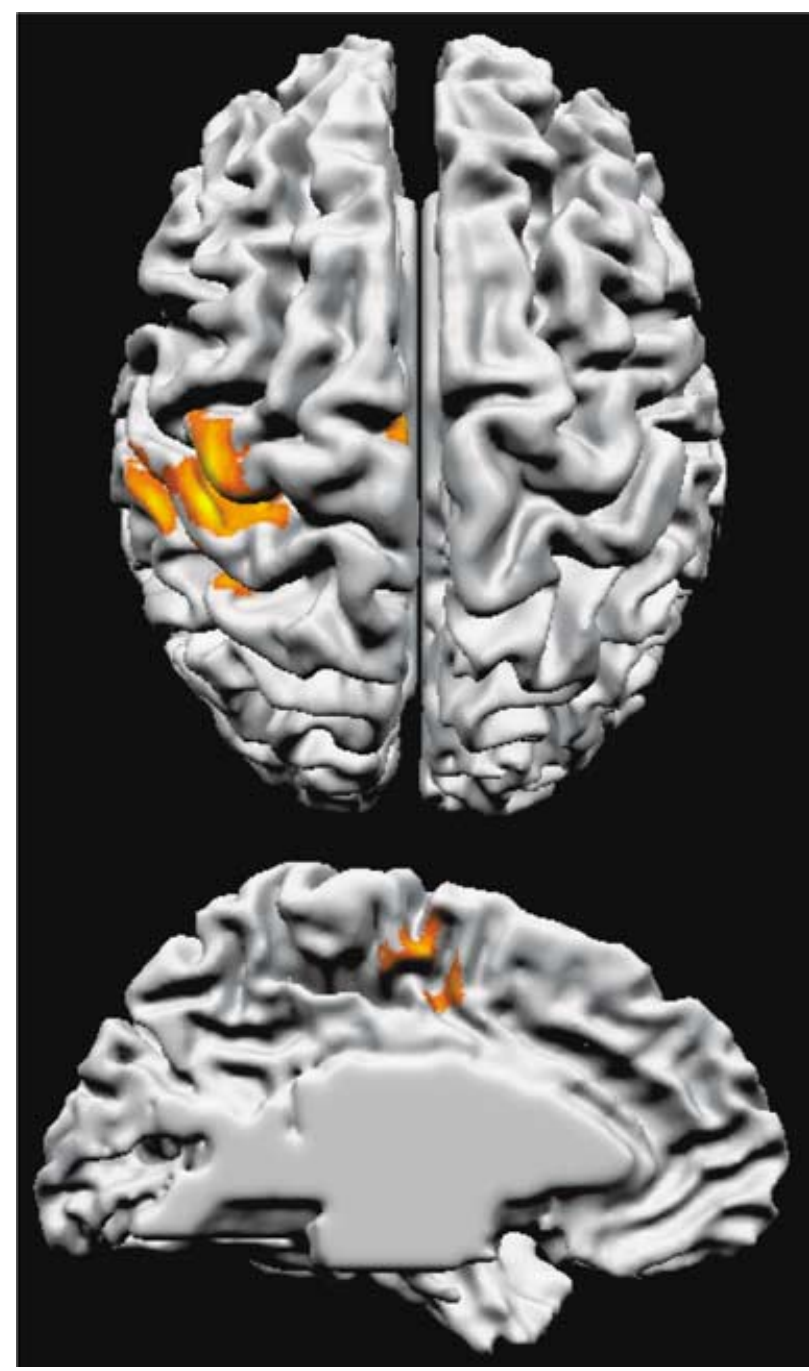

FIG. 9. Brain regions with significantly stronger BOLD MRI activations (11, subjects, $P<0.01$, corrected) evoked by suprathreshold rTMS as compared to subthreshold rTMS projected onto the dorso-lateral surface of a reference brain (MNI). More pronounced suprathreshold rTMS effects were detected in the left M1/S1, PMd, and postcentral sulcus. Medially, stronger activations were found in the SMA and dorsal cingulate gyrus. No significant differences were detected in the auditory cortical and subcortical regions. L, left; R, right.

local GABAergic synapses (Ziemann et al., 1996, 1998; Di Lazzaro et al., 2000). Thus, if subthreshold pulses induce a trans-synaptic excitation of cortical neurons, then why is this not reflected in a BOLD MRI response?
At present, we can only speculate on the possible answers to the above question. Explanations proposed so far range from insufficient sensitivity for the detection of subtle BOLD MRI signal changes at the site of stimulation to a cancellation of inhibitory and excitatory processes that lead to a negligible net synaptic activity with no haemodynamic output, and the occurrence of altered haemodynamic response characteristics that may not be detected by using common response functions (Siebner et al., 2003b). Although we did not test for alternative haemodynamic responses in the stimulated M1/S1, the temporal profile of BOLD MRI signal increases in remote motor regions was in excellent agreement with a large number of preceding fMRI motor studies. We therefore favour a model in which the local haemodynamic changes that are evoked at the site of stimulation by subthreshold rTMS do not exceed the background physiological 'noise' level. Similar ideas were recently reported in a combined SPECT-TMS study (Okabe et al., 2003). Despite widespread activity changes in remote motor regions, no evidence was found for local responses during $1 \mathrm{~Hz}$ rTMS just above AMT. It is also interesting to note that a similar lack of BOLD MRI activations at the site of stimulation was reported after TMS over the lateral premotor cortex (Baudewig et al., 2001; Kemna \& Gembris, 2003) and the postcentral region (Kemna \& Gembris, 2003). In addition, small but non-significant activity increases in M1 were reported in a recent PET-TMS study despite widespread activations in other connected areas (Chouinard et al., 2003).

A second question related to subthreshold rTMS concerns the mechanism that activates areas distant from the site of stimulation. We have argued that such activations are likely to reflect changes in synaptic input from M1/S1, at least for motor-related areas. However, if a subthreshold stimulus does not evoke activity in the corticospinal output from M1, it remains questionable if it can directly activate cortico-cortical or cortico-subcortical pathways. The fact that the presumed transcallosal output from one motor cortex to the other usually has a higher threshold than the corticospinal output (Ferbert et al., 1992) suggests that the answer is negative. We therefore propose that remote motor activations result from a change in the general pattern of ongoing physiological activity in the motor system, which is initiated by a TMS-induced alteration of the cortical excitability within M1/S1. This would be consistent with previous findings of remote excitability changes in response to subthreshold rTMS of the premotor cortex (Gerschlager et al., 2001; Munchau et al., 2002; Rizzo et al., 2003). At low stimulation intensities and short pulse trains the expected small change in the overall neural activity is probably unable to reach the threshold for local haemodynamic increases, which would also be in line with suggested non-linearities of neurovascular coupling (Mathiesen et al., 1998; Lauritzen, 2001). Nevertheless, rTMS at subthreshold intensity might still be able to alter the efferent function of M1 to such a degree that it induces changes in blood flow or BOLD MRI signal strength in directly connected brain regions.

TABLE 3. Differences in activation between rTMS conditions

\begin{tabular}{|c|c|c|c|c|c|}
\hline \multirow[b]{2}{*}{ Anatomical/functional location } & \multicolumn{3}{|c|}{ Talairach coordinates* } & \multirow{2}{*}{$\begin{array}{l}\text { Peak }^{\dagger} \\
\text { activation } \\
t \text {-value }\end{array}$} & \multirow{2}{*}{$\begin{array}{l}\text { Volume } \\
\left(\mathrm{mm}^{3}\right)\end{array}$} \\
\hline & $x$ & $y$ & $z$ & & \\
\hline Left central sulcus (M1/S1) & -36 & -26 & 58 & 8.79 & 1371 \\
\hline Left precentral sulcus (PMd) & -28 & -14 & 65 & 4.88 & 158 \\
\hline Left postcentral gyrus & -51 & -29 & 47 & 6.57 & 1153 \\
\hline Supplementary motor area (SMA) & 0 & -11 & 57 & 6.42 & 669 \\
\hline Cingulate motor area (CMA) & -3 & -9 & 47 & 6.43 & 887 \\
\hline Right cerebellar hemisphere & 11 & -45 & -13 & 6.12 & 166 \\
\hline
\end{tabular}

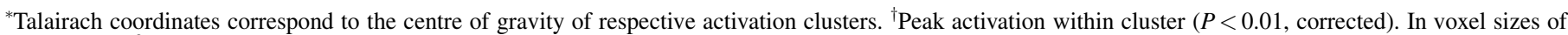
$1 \times 1 \times 1 \mathrm{~mm}^{3}$. 
It should be noted that previous PET-TMS studies have reported strong effects at the site of stimulation when using longer stimulation periods of up to $30 \mathrm{~min}$ (Siebner et al., 2000, 2001a,b, 2003a,c; Lee et al., 2003). Thus, the absence of a BOLD effect to a brief period of subthreshold rTMS seems to indicate a dose-dependency that leads to rather different cortical (and haemodynamic) effects for prolonged stimulation as compared to a short series of stimuli. Further work is needed to address this problem.

Extending previous PET and fMRI studies, both supra- and subthreshold rTMS of the motor cortex evoked localized activity changes in subcortical structures such as the ipsilateral ventro-lateral thalamus and postcommissural portion of the putamen. Less consistent activity was found in the contralateral thalamus and putamen. These subcortical structures are known to be intimately linked to the sensorimotor cortex (Parent \& Hazrati, 1995). The location of thalamic activity corresponded well with previously described motor activity of the thalamus (Lehericy et al., 1998). We found consistent activation in the ventro-lateral nucleus, with some degree of overlap with the ventral posterolateral (VPL) and posteromedial (VPM) nucleus. A possible explanation may result from the fact that rTMS not only activates thalamocortical motor regions related to limb movement (VPL), but also leads to processing of somatosensory information evoked by the stimulated sensation of both the limbs (VPL) and head (VPM). Activity in the basal ganglia was predominantly found in the postcommissural part of the putamen again known to receive strong sensorimotor projections (Parent \& Hazrati, 1995). Involvement of this location is largely in agreement with previous fMRI investigations on basal ganglia activity (Lehericy et al., 1998; Scholz et al., 2000; Gerardin et al., 2003).

Especially during suprathreshold rTMS, several subjects reported some degree of discomfort from the pressure of the vibrating TMS coil on the head. Activations in the anterior insula were only seen during suprathreshold stimulation and may represent neural processing of this unpleasant input. This interpretation is in accordance with Siebner et al. (2001b) who found a linear activity increase in the anterior insular cortex with the frequency of rTMS of the left M1/S1.

\section{Auditory system}

Pronounced activations in the superior temporal gyrus and sulcus and TTG bilaterally were found in all conditions, similar to previous reports using either PET (Siebner et al., 1999) or fMRI (Bohning et al., 1999, 2000b; Baudewig et al., 2001; Nahas et al., 2001). They were primarily located in the upper posterior section of the TTG and superior temporal gyrus in agreement with other recent studies (Bilecen et al., 2002; Brechmann et al., 2002; Hugdahl et al., 2003). The activations extended into the planum temporale and inferior frontal gyrus (putative PMv) as observed for processing of loud stimuli (Bilecen et al., 2002; Brechmann et al., 2002).

Despite a lack of statistical significance, activations in the auditory system tended to be stronger during acoustically triggered finger movements than during sub- or suprathreshold rTMS. In view of studies reporting an intensity-dependent BOLD response to sound in the auditory cortex (Jancke et al., 1998; Bilecen et al., 2002; Brechmann et al., 2002; Lasota et al., 2003), this result is surprising given the substantial differences in sound pressure between the two rTMS conditions and the low-level stimulation used for acoustic triggering. However, inside the static magnetic field of a 3T MRI system, the much enhanced noise of a coil discharge may lead to a ceiling effect in terms of the BOLD response. Thus, a more likely possibility is that auditory activations during voluntary movement were boosted by attentional factors as subjects had to attend to stimulation epochs in order to identify the acoustic trigger and carry out the task, whereas during rTMS subjects were only instructed not to move. In fact, several reports observed substantial attentional modulation of cortical auditory activity. For example, activity along the middle temporal gyri and superior temporal gyrus increased during attention to sounds and words as compared to passive listening (Hugdahl et al., 2003). Attention also modulated the auditory activity as reflected in the early and late components of event-related potentials and magnetic fields (Arthur et al., 1991; Rif et al., 1991). In addition, foreground-background decomposition of different auditory patterns, as presumably also required for a distinction of the TMS clicks from the background noise of the scanner, has been reported to evoke stronger auditory activations than the mere listening to sound patterns above background noise (Brechmann et al., 2002).

Stimulation of the auditory cortex was accompanied by increased synaptic activity in the bilateral inferior colliculi and MGN. These activations within the auditory system are convincingly explained by the click sounds of the discharging TMS coil, which were substantially amplified within the scanner. The MGN reflects the auditory thalamic relay and projects into the primary auditory cortex via the auditory radiation. Although the location and spatial extent of the MGN can vary considerably in stereotaxic space (Rademacher et al., 2002), it was clearly distinguishable from the motor nuclei of the thalamus. There was some degree of inter-individual variability between subjects and often the activations in the inferior colliculus and MGN formed into one cluster. This is presumably due to the relatively low spatial resolution in comparison to the small size of these structures.

\section{Technical considerations}

Although we were able to monitor EMG activity during MRI, this was only accomplished for the primary target muscle of the contralateral hand. We therefore cannot fully exclude the occurrence of myographic activity in adjacent hand or limb muscles that may have contributed to the observed results. Given the relatively low stimulation intensity for subthreshold rTMS, however, it seems unlikely that spread of activation to other hand muscles or even proximal limb muscles took place. This is supported by the absence of significant signal changes in the stimulated M1/S1 during subthreshold rTMS. Processing of the EMG for a reduction of EPI-related artefacts preserved the temporal resolution of the tracings and therefore allowed for a reliable detection of peripheral muscle responses but also precluded a quantitative analysis of the amplitude information.

We are confident that our setup for combined TMS-fMRI properly targeted the left M1/S1 even though we did not use stereotaxic positioning devices. In fact, previous work demonstrated a good correspondence of the optimal scalp position for TMS of the contralateral hand with respective activation clusters for hand movements (Macdonnell et al., 1999; Siebner et al., 2001b; Herwig et al., 2002). In the future, the use of recently introduced MR-guided coil-positioning devices (Bohning et al., 2003) will facilitate accurate coil placement. This will be even more important for brain regions other than M1/S1, for example the prefrontal cortex, which cannot easily be located using TMS.

\section{Concluding remarks}

This work demonstrates the feasibility of combined rTMS-fMRI at a field strength of 3T. Both supra- and subthreshold rTMS of the primary sensorimotor cortex elicited a distinct network of cortical and subcortical motor pathways and, furthermore, unravelled a pronounced involvement of the auditory system. These latter findings emphasize the importance of experimental designs that allow for a control of confounding influences from such undesired stimulations. A comparison of activations obtained for the two rTMS conditions support the 
notion that re-afferent feedback from evoked movements represents the dominant input to the primary motor cortex. The absence of BOLD MRI changes in M1 during subthreshold stimulation may be explained by the fact that the associated alterations of the cortical excitability does not cause local haemodynamic changes that exceed the background physiological noise. Nevertheless, the altered activity patterns in M1/S1 seem to be sufficiently strong to elicit activations of remote motor areas via synaptic connections. The observation of respective BOLD MRI activations confirms the capability of rTMS to target both local and distant brain regions as constituents of a tightly connected cortical and subcortical network.

\section{Acknowledgements}

SB (DFG GK-GRK 632/1-00) and HRS (DFG SI 738/1) are grateful for financial support from the Deutsche Forschungsgemeinschaft. The authors would like to acknowledge the invaluable support from Anthony Thomas, Stefan Cohrs, Lutz Präkelt, and Kurt Böhm. They thank John R. Hernshey and Anders A. Baumann for their assistance on earlier versions of the manuscript.

\section{Abbreviations}

AMT, active motor threshold; BOLD, blood oxygenation level dependent; CMA, cingulate motor area; EMG, electromyography; EPI, echo-planar imaging; fMRI, functional magnetic resonance imaging; M1, primary motor cortex; M1/S1, primary sensorimotor cortex; MGN, medial geniculate nucleus; PMd, dorsal premotor cortex; RF, radiofrequency; RMT, resting motor threshold; ROI, region-of-interest; rTMS, repetitive TMS; SMA, supplementary motor cortex; TMS, transcranial magnetic stimulation; TTG, transverse temporal gyrus; VLN, ventro-lateral nucleus.

\section{References}

Arthur, D.L., Lewis, P.S., Medvick, P.A. \& Flynn, E.R. (1991) A neuromagnetic study of selective auditory attention. Electroencephalogr. Clin. Neurophysiol., 78, 348-360.

Baudewig, J., Dechent, P., Merboldt, K.D. \& Frahm, F. (2003) Thresholding in correlation analysis of magnetic resonance functional neuroimaging. Magn. Reson. Imaging, 21, 1121-1130.

Baudewig, J., Paulus, W. \& Frahm, J. (2000) Artefacts caused by transcranial magnetic stimulation coils and EEG electrodes in T2*-weighted echo-planar imaging. Magn. Reson. Imaging, 18, 479-484.

Baudewig, J., Siebner, H.R., Bestmann, S., Tergau, F., Tings, T., Paulus, W. \& Frahm, J. (2001) Functional MRI of cortical activations induced by transcranial magnetic stimulation (TMS). Neuroreport, 12, 3543-3548.

Bäumer, T., Lange, R., Liepert, J., Weiller, C., Siebner, H.R., Rothwell, J.C. \& Munchau, A. (2003) Repeated premotor rTMS leads to cumulative plastic changes of motor cortex excitability in humans. Neuroimage, 20, 550-560.

Bestmann, S., Baudewig, J. \& Frahm, J. (2003a) On the synchronization of transcranial magnetic stimulation and functional echo-planar imaging. $J$. Magn. Reson. Imaging, 17, 309-316.

Bestmann, S., Baudewig, J., Siebner, H.R., Rothwell, J.C. \& Frahm, J. (2003b) Subthreshold high-frequency TMS of human primary motor cortex modulates interconnected frontal motor areas as detected by interleaved fMRITMS. Neuroimage, 20, 1685-1696.

Bestmann, S., Baudewig, J., Siebner, H.R., Rothwell, J.C. \& Frahm, J. (2003c) Is functional magnetic resonance imaging capable of mapping transcranial magnetic cortex stimulation? Clin. Neurophysiol. Suppl., 56, 55-62.

Bestmann, S., Siebner, H.R., Modugno, N., Amassian, V. \& Rothwell, J.C. (2004) Inhibitory interaction between pairs of subthreshold conditioning stimuli in the human motor cortex. Clin. Neurophysiol., in press.

Bilecen, D., Seifritz, E., Scheffler, K., Henning, J. \& Schulte, A.C. (2002) Amplitopicity of the human auditory cortex: an fMRI study. Neuroimage, 17, 710-718.

Bohning, D.E., Denslow, S., Bohning, P.A., Walker, J.A. \& George, M.S. (2003) A TMS coil positioning/holding device for MR image-guided TMS interleaved with fMRI. Clin. Neurophysiol., 114, 1997-2223.

Bohning, D.E., Shastri, A., McConnell, K.A., Nahas, Z., Lorberbaum, J.P., Roberts, D.R., Teneback, C., Vincent, D.J. \& George, M.S. (1999) A combined TMS/fMRI study of intensity-dependent TMS over motor cortex. Biol. Psychiatry, 45, 385-394.
Bohning, D.E., Shastri, A., McGavin, L., McConnell, K.A., Nahas, Z., Lorberbaum, J.P., Roberts, D.R. \& George, M.S. (2000a) Motor cortex brain activity induced by $1-\mathrm{Hz}$ Transcranial Magnetic Stimulation is similar in location and level to that for volitional movement. Invest. Radiol., 11, 676-683.

Bohning, D.E., Shastri, A., Nahas, Z., Lorberbaum, J.P., Andersen, S.W., Dannels, W.R., Haxthausen, E.U., Vincent, D.J. \& George, M.S. (1998) Echoplanar BOLD fMRI of brain activation induced by concurrent transcranial magnetic stimulation. Invest. Radiol., 33, 336-340.

Bohning, D.E., Shastri, A., Wassermann, E.M., Ziemann, U., Lorberbaum, J.P., Nahas, Z., Lomarev, M.P. \& George, M.S. (2000b) BOLD-fMRI response to single-pulse transcranial magnetic stimulation (TMS). J. Magn. Reson. Imaging, 11, 569-574.

Boynton, G.M., Engel, S.A., Glover, G.H. \& Heeger, D.J. (1996) Linear systems analysis of functional magnetic resonance imaging in human V1. J. Neurosci., 16, 4207-4221.

Brechmann, A., Baumgart, F. \& Scheich, H. (2002) Sound-level-dependent representation of frequency modulations in human auditory cortex: a lownoise fMRI study. J. Neurophysiol., 87, 423-433.

Chouinard, P.A., Van Der Werf, Y.D., Leonard, G. \& Paus, T. (2003) Modulating neural networks with transcranial magnetic stimulation applied over the dorsal premotor and primary motor cortices. J. Neurophysiol., 90, 1071-1083.

Cowey, A. \& Walsh, V. (2001) Tickling the brain: studying visual sensation, perception and cognition by transcranial magnetic stimulation. Prog. Brain Res., 134, 411-425.

Di Lazzaro, V., Oliviero, A., Mazzone, P., Pilato, F., Saturno, E., Dileone, M., Insola, A., Tonali, P.A. \& Rothwell, J.C. (2002) Short-term reduction of intracortical inhibition in the human motor cortex induced by repetitive transcranial magnetic stimulation. Exp. Brain Res., 147, 108-113.

Di Lazzaro, V., Oliviero, A., Meglio, M., Cioni, B., Tamburrini, G., Tonali, P. \& Rothwell, J.C. (2000) Direct demonstration of the effect of lorazepam on the excitability of the human motor cortex. Clin. Neurophysiol., 111, 794-799.

Di Lazzaro, V., Restuccia, D., Oliviero, A., Profice, P., Ferrara, L., Insola, A., Mazzone, P., Tonali, P. \& Rothwell, J.C. (1998) Magnetic transcranial stimulation at intensities below active motor threshold activates intracortical inhibitory circuits. Exp. Brain Res., 119, 265-268.

Ferbert, A., Priori, A., Rothwell, J.C., Day, B.L., Colebatch, J.G. \& Marsden, C.D. (1992) Interhemispheric inhibition of the human motor cortex. $J$. Physiol. (Lond.), 453, 525-546.

Fink, G.R., Frackowiak, R.S., Pietrzyk, U. \& Passingham, R.E. (1997) Multiple nonprimary motor areas in the human cortex. J. Neurophysiol., 77, 2164 2174.

Fisher, R.J., Nakamura, Y., Bestmann, S., Rothwell, J.C. \& Bostock, H. (2002) Two phases of intracortical inhibition revealed by transcranial magnetic threshold tracking. Exp. Brain Res., 143, 240-248.

Fox, P., Ingham, R., George, M.S., Mayberg, H., Ingham, J., Roby, J., Martin, C. \& Jerabek, P. (1997) Imaging human intra-cerebral connectivity by PET during TMS. Neuroreport, $\mathbf{8}, 2787-2791$.

Gerardin, E., Lehericy, S., Pochon, J.B., Tezenas du Montcel, S., Mangin, J.F., Poupon, F., Agid, Y., Le Bihan, D. \& Marsault, C. (2003) Foot, hand, face and eye representation in the human striatum. Cereb. Cortex, 13, 162-169.

Gerschlager, W., Siebner, H.R. \& Rothwell, J.C. (2001) Decreased corticospinal excitability after subthreshold $1 \mathrm{~Hz}$ rTMS over lateral premotor cortex. Neurology, 57, 449-455

Geyer, S., Matelli, M., Luppino, G. \& Zilles, K. (2000) Functional neuroanatomy of the primate isocortical motor system. Anat. Embryol. (Berl.), 202, 443-474.

Grezes, J. \& Decety, J. (2001) Functional anatomy of execution, mental simulation, observation, and verb generation of actions: a meta-analysis. Hum. Brain Mapp., 12, 1-19.

Herwig, U., Kolbel, K., Wunderlich, A.P., Thielscher, A., von Tiesenhausen, C., Spitzer, M. \& Schonfeldt-Lecuona, C. (2002) Spatial congruence of neuronavigated transcranial magnetic stimulation and functional neuroimaging. Clin. Neurophysiol., 113, 462-468.

Hlustik, P., Solodkin, A., Gullapalli, R.P., Noll, D.C. \& Small, S.L. (2002) Functional lateralization of the human premotor cortex during sequential movements. Brain Cogn., 49, 54-62.

Hugdahl, K., Thomsen, T., Ersland, L., Morten Rimol, L. \& Niemi, J. (2003) The effects of attention on speech perception: an fMRI study. Brain Lang., 85, 37-48.

Ilmoniemi, R.J., Ruohonen, J. \& Karhu, J. (1999) Transcranial magnetic stimulation-a new tool for functional imaging of the brain. Crit. Rev. Biomed. Eng., 27, 241-284. 
Jancke, L., Shah, N.J., Posse, S., Grosse-Ryuken, M. \& Muller-Gartner, H.W. (1998) Intensity coding of auditory stimuli: an fMRI study. Neuropsychologia, 36, 875-883.

Kemna, L.J. \& Gembris, D. (2003) Repetitive transcranial magnetic stimulation indices different responses in different cortical areas: a functional magnetic resonance study in humans. Neurosci. Lett., 336, 85-88.

Kujirai, T., Caramia, M.D., Rothwell, J.C., Day, B.L., Thompson, P.D., Ferbert, A., Wroe, S., Asselman, P. \& Marsden, C.D. (1993) Corticocortical inhibition in human motor cortex. J. Physiol. (Lond.), 471, 501-519.

Lasota, K.J., Ulmer, J.L., Firszt, J.B., Biswal, B.B., Daniels, D.L. \& Prost, R.W. (2003) Intensity-dependent activation of the primary auditory cortex in functional magnetic resonance imaging. J. Comput. Assist. Tomogr., 27, 213-218.

Laufs, H., Kleinschmidt, A., Beyerle, A., Eger, E., Salek-Haddadi, A., Preibisch, C. \& Krakow, K. (2003) EEG-correlated fMRI of human alpha activity. Neuroimage, 19, 1463-1476.

Lauritzen, M. (2001) Relationship of spikes, synaptic activity, and local changes of cerebral blood flow. J. Cereb. Blood Flow. Metab., 21, 1367-1383.

Lee, L., Siebner, H.R., Rowe, J.B., Rizzo, V., Rothwell, J.C., Frackowiak, R.S. \& Friston, K.J. (2003) Acute remapping within the motor system induced by low-frequency repetitive transcranial magnetic stimulation. J. Neurosci., 23, $5308-5318$.

Lehericy, S., van de Moortele, P.F., Lobel, E., Paradis, A.L., Vidailhet, M., Frouin, V., Neveu, P., Agid, Y., Marsault, C. \& Le Bihan, D. (1998) Somatotopical organization of striatal activation during finger and toe movement: a 3-T functional magnetic resonance imaging study. Ann. Neurol., 44, 398-404.

Macdonell, R.A., Jackson, G.D., Curatolo, J.M., Abbott, D.F., Berkovic, S.F., Carey, L.M., Syngeniotin, A., Fabinyi, G.C. \& Scheffer, I.E. (1999) Motor cortex localization using functional MRI and transcranial magnetic stimulation. Neurology, 53, 1462-1467.

Mathiesen, C., Caesar, K., Akgoren, N. \& Lauritzen, M. (1998) Modification of activity-dependent increases of cerebral blood flow by excitatory synaptic activity and spikes in rat cerebellar cortex. J. Physiol. (Lond.), 512, 555-566.

Munchau, A., Bloem, B.R., Irlbacher, K., Trimble, M.R. \& Rothwell, J.C. (2002) Functional connectivity of human premotor and motor cortex explored with repetitive transcranial magnetic stimulation. J. Neurosci., 22, 554-561.

Nahas, Z., Lomarev, M., Roberts, D.R., Shastri, A., Lorberbaum, J.P., Teneback, C., McConnell, K., Vincent, D.J., Li, X., George, M.S. \& Bohning, D.E. (2001) Unilateral left prefrontal transcranial magnetic stimulation (TMS) produces intensity-dependent bilateral effects as measured by interleaved BOLD fMRI. Biol. Psychiatry, 50, 712-720.

Ogawa, S., Lee, T.M., Kay, A.R. \& Tank, D.W. (1990) Brain magnetic resonance imaging with contrast dependent on blood oxygenation. Proc. Natl Acad. Sci. USA, 87, 9868-9872.

Okabe, S., Hanajima, R., Ohnishi, T., Nishikawa, M., Imabayashi, E., Takano, H., Kawachi, T., Matsuda, H., Shiio, Y., Iwata, N.K., Furubayashi, T., Terao, Y. \& Ugawa, Y. (2003) Functional connectivity revealed by single-photon emission computed tomography (SPECT) during repetitive transcranial magnetic stimulation (rTMS) of the motor cortex. Clin. Neurophysiol., 114, 450-457.

Oldfield, R.C. (1971) The assessment and analysis of handedness: the Edinburgh inventory. Neuropsychologia, 9, 97-113.

Oliviero, A., Strens, L.H., Di Lazzaro, V., Tonali, P.A. \& Brown, P. (2003) Persistent effects of high frequency repetitive TMS on the coupling between motor areas in the human. Exp. Brain Res., 149, 107-113.

Parent, A. \& Hazrati, L.N. (1995) Functional anatomy of the basal ganglia. I. The cortico-basal ganglia-thalamo-cortical loop. Brain Res. Brain Res. Rev., 20, 91-127.

Pascual-Leone, A., Valls-Sole, J., Wassermann, E.M. \& Hallett, M. (1994) Responses to rapid-rate transcranial magnetic stimulation of the human motor cortex. Brain, 117, 847-858.

Paus, T. (1999) Imaging the brain before, during, and after transcranial magnetic stimulation. Neuropsychologia, 37, 219-224.

Paus, T. (2000) Functional anatomy of arousal and attention systems in the human brain. Prog. Brain Res., 126, 65-77.

Paus, T. (2002) Combination of transcranial magnetic stimulation and brain mapping. In: A. Toga, A. Mazziotta \& J., (Eds), Brain Mapping: the Methods, 2nd Edn. Elsevier, Amsterdam, pp. 691-705.

Paus, T., Castro-Alamancos, M.A. \& Petrides, M. (2001a) Cortico-cortical connectivity of the human mid-dorsolateral frontal cortex and its modulation by repetitive transcranial magnetic stimulation. Eur. J. Neurosci., 14, 1405-1411.

Paus, T., Jech, R., Thompson, C.J., Comeau, R., Peters, T. \& Evans, A.C. (1997) Transcranial magnetic stimulation during positron emission tomography: a new method for studying connectivity of the human cerebral cortex. $J$. Neurosci., 17, 3178-3184.

Paus, T., Jech, R., Thompson, C.J., Comeau, R., Peters, T. \& Evans, A.C. (1998) Dose-dependent reduction of cerebral blood flow during rapid-rate transcranial magnetic stimulation of the human sensorimotor cortex. J. Neurophysiol., 79, 1102-1107.

Paus, T., Sipila, P.K. \& Strafella, A.P. (2001b) Synchronization of neuronal activity in the human primary motor cortex by transcranial magnetic stimulation: an EEG study. J. Neurophysiol., 86, 1983-1990.

Paus, T., Tomaiuolo, F., Otaky, N., MacDonald, D., Petrides, M., Atlas, J., Morris, R. \& Evans, A.C. (1996) Human cingulate and paracingulate sulci: pattern, variability, asymmetry, and probabilistic map. Cereb. Cortex, 6, 207-214

Picard, N. \& Strick, P.L. (1996) Medial wall motor areas: a review of their location and functional activation. Cereb. Cortex, 6, 342-353.

Picard, N. \& Strick, P.L. (2001) Imaging the premotor areas. Curr. Opin. Neurobiol., 11, 663-672.

Rademacher, J., Burgel, U. \& Zilles, K. (2002) Stereotaxic localization, intersubject variability, and interhemispheric differences of the human auditory thalamocortical system. Neuroimage, 17, 142-160.

Rif, J., Hari, R., Hamalainen, M.S. \& Sams, M. (1991) Auditory attention affects two different areas in the human supratemporal cortex. Electroencephalogr. Clin. Neurophysiol., 79, 464-472.

Rizzo, V., Siebner, H.R., Modugno, N., Pesenti, A., Munchau, A., Gerschlager, W., Webb, R.M. \& Rothwell, J.C. (2004) Shaping the excitability of human motor cortex with premotor rTMS. J. Physiol. (Lond.), 554, 483-495.

Rizzolatti, G., Luppino, G. \& Matelli, M. (1996) The classic supplementary motor area is formed by two independent areas. Adv. Neurol., 70, 45-56.

Rossi, S., Pasqualetti, P., Rossini, P.M., Feige, B., Ulivelli, M., Glocker, F.X., Battistini, N., Lucking, C.H. \& Kristeva-Feige, R. (2000) Effects of repetitive transcranial magnetic stimulation on movement-related cortical activity in humans. Cereb. Cortex, 10, 802-808.

Scholz, V.H., Flaherty, A.W., Kraft, E., Keltner, J.R., Kwong, K.K., Chen, Y.I., Rosen, B.R. \& Jenkins, B.G. (2000) Laterality, somatotopy and reproducibility of the basal ganglia and motor cortex during motor tasks. Brain Res., 879, 204-215.

Shastri, A., George, M.S. \& Bohning, D.E. (1999) Performance of a system for interleaving transcranial magnetic stimulation with steady-state magnetic resonance imaging. Electroencephal. Clin. Neurophysiol. Suppl., 51, 55-64.

Siebner, H.R., Filipovic, S.R., Rowe, J.B., Cordivari, C., Gerschlager, W., Rothwell, J.C., Frackowiak, R.S. \& Bhatia, K.P. (2003a) Patients with focal arm dystonia have increased sensitivity to slow-frequency repetitive TMS of the dorsal premotor cortex. Brain, 126, 2710-2725.

Siebner, H.R., Lee, L. \& Bestmann, S. (2003b) Interleaving TMS with functional MRI: Now that it is technically feasible how should it be used? Clin. Neurophysiol., 114, 1997-1999.

Siebner, H., Peller, M., Bartenstein, P., Willoch, F., Rossmeier, C., Schwaiger, M. \& Conrad, B. (2001a) Activation of frontal premotor areas during suprathreshold transcranial magnetic stimulation of the left primary sensorimotor cortex: a glucose metabolic PET study. Hum. Brain Mapp., 12, $157-167$.

Siebner, H.R., Peller, M. \& Lee, L. (2003c) Applications of combined TMSPET studies in clinical and basic research. Clin. Neurophysiol. Suppl., 56, 63-74.

Siebner, H.R., Peller, M., Willoch, F., Auer, C., Bartenstein, P., Drzezga, A., Schwaiger, M. \& Conrad, B. (1999) Imaging functional activation of the auditory cortex during focal repetitive transcranial magnetic stimulation of the primary motor cortex in normal subjects. Neurosci. Lett., 270, $37-40$.

Siebner, H.R., Peller, M., Willoch, F., Minoshima, S., Boecker, H., Auer, C., Drzezga, A., Conrad, B. \& Bartenstein, P. (2000) Lasting cortical activation after repetitive TMS of the motor cortex: a glucose metabolic study. Neurology, 54, 956-963.

Siebner, H.R. \& Rothwell, J.C. (2003) Transcranial magnetic stimulation: new insights into representational cortical plasticity. Exp. Brain Res., 148, 1-16.

Siebner, H.R., Takano, B., Peinemann, A., Schwaiger, M., Conrad, B. \& Drzezga, A. (2001b) Continuous transcranial magnetic stimulation during positron emission tomography: a suitable tool for imaging regional excitability of the human cortex. Neuroimage, 14, 883-890.

Speer, A.M., Willis, M.W., Herscovitch, P., Daube-Witherspoon, M., Repella Shelton, J., Benson, B.E., Post, R.M. \& Wassermann, E.M. (2003a) Intensitydependent regional cerebral blood flow during $1-\mathrm{Hz}$ repetitive transcranial magnetic stimulation (rTMS) in healthy volunteers studied with $\mathrm{H}(2)\left({ }^{15}\right) \mathrm{O}$ positron emission tomography: I. effects of primary motor cortex rTMS. Biol. Psychiatry, 54, 818-825. 
Speer, A.M., Willis, M.W., Herscovitch, P., Daube-Witherspoon, M., Repella Shelton, J., Benson, B.E., Post, R.M. \& Wassermann, E.M. (2003b) Intensitydependent regional cerebral blood flow during $1-\mathrm{Hz}$ repetitive transcranial magnetic stimulation (rTMS) in healthy volunteers studied with $\mathrm{H}\left({ }_{2}\right)\left({ }^{15}\right) \mathrm{O}$ positron emission tomography: II. effects of prefrontal cortex rTMS. Biol. Psychiatry, 54, 826-832.

Strafella, A.P. \& Paus, T. (2001) Cerebral blood-flow changes induced by paired-pulse transcranial magnetic stimulation of the primary motor cortex. J. Neurophysiol., 85, 2624-1629.

Strafella, A.P., Paus, T., Fraraccio, M. \& Dagher, A. (2003) Striatal dopamine release induced by repetitive transcranial magnetic stimulation of the human motor cortex. Brain, 126, 2609-2615.

Strens, L.H., Oliviero, A., Bloem, B.R., Gerschlager, W., Rothwell, J.C. \& Brown, P. (2002) The effects of subthreshold $1 \mathrm{~Hz}$ repetitive TMS on corticocortical and interhemispheric coherence. Clin. Neurophysiol., 113, 1279-1285.

Talairach, J. \& Tournoux, P. (1988) Co-Planar Stereotaxic Atlas of the Human Brain. Thieme, Stuttgart.

Thut, G., Northoff, G., Ives, J.R., Kamitani, Y., Pfennig, A., Kampmann, F., Schomer, D.L. \& Pascual-Leone, A. (2003) Effects of single-pulse transcranial magnetic stimulation (TMS) on functional brain activity: a combined event-related TMS and evoked potential study. Clin. Neurophysiol., 114, 2071-2080.
Walsh, V. \& Cowey, A. (2000) Transcranial magnetic stimulation and cognitive neuroscience. Nature Rev. Neurosci., 1, 73-79.

Wassermann, E.M. (1998) Risk and safety of repetitive transcranial magnetic stimulation: report and suggested guidelines from the International Workshop on the Safety of Repetitive Transcranial Magnetic Stimulation, June 57, 1996. Electroencephalogr. Clin. Neurophysiol., 108, 1-16.

White, L.E., Andrews, T.J., Hulette, C., Richards, A., Groelle, M., Paydarfar, J. \& Purves, D. (1997) Structure of the human sensorimotor system. I: Morphology and cytoarchitecture of the central sulcus. Cereb. Cortex, 7, $18-30$.

Yousry, T.A., Schmid, U.D., Alkadhi, H., Schmidt, D., Peraud, A., Buettner, A. \& Winkler, P. (1997) Localization of the motor hand area to a knob on the precentral gyrus: a new landmark. Brain, 120, 141-157.

Ziemann, U., Lonnecker, S., Steinhoff, B.J. \& Paulus, W. (1996) Effects of antiepileptic drugs on motor cortex excitability in humans: a transcranial magnetic stimulation study. Ann. Neurol., 40, 367-378.

Ziemann, U. \& Rothwell, J.C. (2000) I-waves in motor cortex. J. Clin. Neurophysiol., 17, 397-405.

Ziemann, U., Tergau, F., Wischer, S., Hildebrandt, J. \& Paulus, W. (1998) Pharmacological control of facilitatory I-wave interaction in the human motor cortex. A paired transcranial magnetic stimulation study. Electroencephalogr. Clin. Neurophysiol., 109, 321-330. 